\title{
Block-distributed Gradient Boosted Trees
}

\author{
Theodore Vasiloudis* \\ RISE AI \\ tvas@sics.se
}

\author{
Hyunsu Cho \\ Amazon Web Services \\ chyunsu@amazon.com
}

\author{
Henrik Boström \\ KTH Royal Institute Of Technology \\ bostromh@kth.se
}

\begin{abstract}
The Gradient Boosted Tree (GBT) algorithm is one of the most popular machine learning algorithms used in production, for tasks that include Click-Through Rate (CTR) prediction and learningto-rank. To deal with the massive datasets available today, many distributed GBT methods have been proposed. However, they all assume a row-distributed dataset, addressing scalability only with respect to the number of data points and not the number of features, and increasing communication cost for high-dimensional data.

In order to allow for scalability across both the data point and feature dimensions, and reduce communication cost, we propose block-distributed GBTs. We achieve communication efficiency by making full use of the data sparsity and adapting the Quickscorer algorithm to the block-distributed setting. We evaluate our approach using datasets with millions of features, and demonstrate that we are able to achieve multiple orders of magnitude reduction in communication cost for sparse data, with no loss in accuracy, while providing a more scalable design. As a result, we are able to reduce the training time for high-dimensional data, and allow more cost-effective scale-out without the need for expensive network communication.
\end{abstract}

\section{KEYWORDS}

Gradient Boosted Trees; Distributed Systems; Communication Efficiency; Scalability

\section{ACM Reference Format:}

Theodore Vasiloudis, Hyunsu Cho, and Henrik Boström. 2019. Block-distributed Gradient Boosted Trees. In Proceedings of the 42nd International ACM SIGIR Conference on Research and Development in Information Retrieval (SIGIR '19), Fuly 21-25, 2019, Paris, France. ACM, New York, NY, USA, 4 pages. https://doi.org/10.1145/3331184.3331331

\section{INTRODUCTION}

In some of the most important Information Retrieval (IR) tasks like CTR prediction and learning-to-rank, Gradient Boosted Trees have risen as one of the most effective algorithms, due to their accuracy and scalability $[5,10]$.

For massive amounts of data that are common at web-scale, distributed learning methods have become necessary. In such scenarios, we want to have the best possible utilization of resources and scalability, to minimize the cost of training a new model.

${ }^{*}$ Work performed during an internship at Amazon.

Permission to make digital or hard copies of part or all of this work for personal or classroom use is granted without fee provided that copies are not made or distributed for profit or commercial advantage and that copies bear this notice and the full citation on the first page. Copyrights for third-party components of this work must be honored

For all other uses, contact the owner/author(s).

SIGIR '19, July 21-25, 2019, Paris, France

(C) 2019 Copyright held by the owner/author(s)

ACM ISBN 978-1-4503-6172-9/19/07.

https://doi.org/10.1145/3331184.3331331
Currently, distributed GBT algorithms [3, 6, 7] use row distribution, in which every worker node processes a horizontal slice of the data matrix, i.e. a subset of the data points with all feature values. Row distribution enables scalability only with respect to the number of data points. As a result, for high-dimensional data with millions of features, existing algorithms require large amounts of memory, which we can only remedy by adding worker nodes to the cluster, further increasing the amount of network communication.

In addition, the existing algorithms use dense communication to aggregate gradient histograms between the workers. Algorithms like XGBoost [3] and LightGBM [7] use all-reduce [14] or similar approaches, where we would need to communicate millions of histograms, one per feature, regardless of whether a feature is present in a data partition or not. As a result, the communication cost may become impractical for sparse, high-dimensional data. The issue is more pressing for multi-tenant clusters or cloud environments commonly used for training [12], since the GBT training job needs to compete with other running jobs for network bandwidth.

To address these issues, we introduce a block-distributed GBT algorithm that partitions the training data in both the row and column dimensions. The new algorithm adds a second dimension along which we can scale-out our learning and uses a sparse gradient histogram representation to minimize network communication. Unlike existing algorithms for feature-parallel training [3, 7], our proposed algorithm does not assume that the complete dataset would fit into the main memory of each worker node.

Block distribution introduces a new algorithmic challenge: the worker nodes no longer have complete access to all feature values. The regular method of computing prediction outputs for decision trees, top-to-bottom traversal, no longer works, because only a subset of feature values are visible to each worker. We develop a novel use of the Quickscorer algorithm $[4,11]$ for block-distributed prediction, to make prediction using parts of each data point feasible and efficient.

However, the new method for calculating tree outputs introduces additional communication steps in the training pipeline. Careful design is therefore necessary to offset the cost. To that end, our proposed algorithm uses the parameter server [8,9] to efficiently transmit sparse data, unlike the dense communication used by current methods $[3,6,7]$. Although the use of the parameter server adds overhead for dense, low-dimensional data, compared to allreduce, it results in orders of magnitude reduced communication cost for sparse, high-dimensional data.

To summarize, our contributions are the following:

- We propose the first block-distributed GBT algorithm, allowing for scalability across both the data point and feature dimensions.

- We provide an experimental evaluation on datasets with up to millions of features. We illustrate the advantages and limitations of the approach in sparse and dense settings. 
- We demonstrate orders of magnitude improved communication cost by taking full advantage of data sparsity during training.

\section{BACKGROUND}

In this section we will provide a brief introduction to gradient boosting, the relevant parts of row-distributed Gradient Boosted Tree learning and related work. We refer the reader to [1] for an in-depth survey of gradient boosting.

\subsection{Gradient Boosted Trees}

GBT learning algorithms all follow a similar base algorithm. At each iteration, we first make predictions on the training data using the current ensemble. We then get the gradients for each data point according to our loss function, and use those gradients to determine the gradient histograms for every feature, at every leaf. Finally, we grow the tree by finding for each leaf its optimal split point.

The gradient histograms for a leaf-feature combination, determine what would be the gradient distribution for the left and right child of that leaf, if we were to split the leaf using that feature, for every possible value of the feature. Our aim as we optimize the tree is to select splits that maximize the gain made possible by splitting a leaf, in terms of a loss function, like squared loss [1].

Doing this for every possible feature and value quickly becomes untenable for real-valued features, so most algorithms make use of quantized histograms [3]. We determine a number of buckets $B$, create empirical histograms for each feature to determine candidate split points, and iterate through this pre-determined number of split points for every feature when evaluating splits. We can then select the optimal split for each leaf by simply sorting the feature-value combinations.

\subsection{Parameter Server \& All-Reduce}

In distributed machine learning there are two dominant communication patterns, each with its merits and drawbacks. The all-reduce primitive performs an aggregation across the cluster, at the end of which, every worker in the cluster ends up with the same copy of the aggregated data. Algorithms like a binomial tree all-reduce [14] are used to minimize communication. However, all-reduce uses dense communication and requires the byte size of the parameters being communicated to be known in advance, resulting in redundancies in the case of sparse data.

The parameter server architecture [9] on the other hand has machines play different roles. Workers are responsible for computation and communicate with servers responsible for storing and updating parameters. The parameter server is more flexible than an all-reduce approach, at the cost of added complexity.

\subsection{Row-distributed Gradient Boosted Trees}

In the row-distributed setting each worker gets a horizontal partition of the data, i.e. a subset of the data points with all features included, makes predictions, and calculates the local gradient histograms for its partition. These gradients need then to be aggregated with every other worker so that they all end up with the same combined gradient histograms. Once the gradients have been communicated, each worker can use its local copy to find the optimal splits, and update the model.
Row-distributed methods utilize dense communication: The algorithms will, for every leaf and every feature, communicate $B$ values, where $B$ the maximum bucket count, regardless of the actual number of unique values for that leaf-feature combination.

For example, assume a continuous feature that we quantize to 255 buckets, which is the default choice for XGBoost. As we grow the tree and partition the dataset into smaller groups, it becomes more likely that many of these 255 value ranges will not be represented in the partition present in the leaf. However, the all-reduce methods require the number of values to be communicated to be known in advance, so we will end up communicating for every leaf the full 255 values, despite the fact that many of those values will be zero. This problem is further magnified in sparse datasets that are common in CTR and learning-to-rank tasks.

Through our use of sparse histogram representations we aim to overcome this shortcoming, by communicating for each leaf only the strictly necessary values in a histogram, vastly improving the communication cost for sparse datasets.

The XGBoost [3] and LightGBM [7] systems provide ways to distribute the computation across only either the data point or feature dimensions, but for the feature-distributed computation they assume that the complete dataset fits in the memory of each worker. CatBoost [13] focused on efficient training for categorical features. Finally, DimBoost [6] was the first paper to use the parameter server for GBTs and brought a number of improvements, such as gradient compression, and a load-balanced split selection algorithm, however it also uses row distribution and dense communication.

\section{METHOD}

In this section we provide an overview of the methods we developed to make block-distributed GBT training possible, and the optimizations that make the process efficient. We use the parameter server for network communication. In this setting, the worker nodes have access to the data and perform computations, like calculating the local gradient histograms, while the servers are responsible for aggregating the parameters sent by the workers.

\subsection{Block-distributed prediction}

In the block-distributed setting every worker has access to a block of the data matrix, that is, data are sliced both horizontally and vertically, and one data block is assigned to each worker.

In order to determine the exit leaf $e$, that is, the leaf where a data point ends up in when dropped down a tree, we will need to communicate between the workers that share different parts of the same rows in the dataset. To minimize the communication cost and achieve provably correct predictions we make use of the Quickscorer (QS) [11] algorithm that we adapt to work in the blockdistributed setting. We briefly describe the algorithm here and refer the reader to the original text for an in-depth description.

Quickscorer overview. The QS algorithm works by assigning a bitstring to every node in a tree, that indicates which leafs would be removed from the set of candidate exit leafs whenever the condition in the node is evaluated as false. Every internal node in the tree is assigned a bitstring of length $|L|$, where $L$ is the set of leaves in the tree. The bitstring of a node has zeros at the bits corresponding 
to the leaves that would become impossible to reach if the node's condition evaluates to false, and ones everywhere else.

We assume that there exists an oracle FindFalse that, given an input $\mathbf{x}$, returns the set of all internal nodes whose conditions evaluate to false for $\mathbf{x}$, without the need to evaluate all the associated test conditions. Once we have that set, we initialize bit vector $\mathbf{v}$ with all 1's and then update $\mathbf{v}$ by performing bitwise-AND with every bitstring in the set. Lucchese et al. [11] prove that the exit leaf $e$ corresponds to the left-most bit set to 1 in the updated $\mathbf{v}$.

Block-distributed Quickscorer. We adapt the above algorithm to work in the case where each worker only has access to a subset of the features for each data point. To fully determine the exit node $e$ for a single data point $\mathbf{x}$, we need to combine the predictions from multiple workers, that hold the different parts of the data point. Since each worker only has a range of features available, it can only evaluate the conditions for the features it has available. We use Quickscorer to only communicate prediction bitstrings, achieving significant communication savings compared to communicating complete models or data points.

Let $N$ be the set of internal nodes in a tree. Each internal node contains a condition of the form $\mathrm{x}\left[f_{n}\right] \leq \gamma$, where $f_{n}$ the selected feature for the node and $\gamma$ the threshold. Let $W$ be the set of workers, and $S$ the set of servers. Each worker $w$ will only be able to evaluate the condition for a subset of the nodes $N_{w}$, where $\cup_{w} N_{w}=N$.

For example, say we have ten features in the data, and three of those, $\left\{f_{1}, f_{2}, f_{8}\right\}$, are conditions in the tree. Let worker 1 be responsible for features 1-5 and worker 2 responsible for features $6-$ 10. Then to fully determine the exit leaf for a data point $\mathbf{x}$ we would evaluate conditions $\left\{f_{1}, f_{2}\right\}$ on worker 1 and condition $\left\{f_{8}\right\}$ on worker 2. Each worker then pushes their partial bitstrings $\left\{\mathbf{v}_{1}, \mathbf{v}_{2}\right\}$ to a server, which performs the bit-wise AND operation $\mathbf{v}=\mathbf{v}_{1} \wedge \mathbf{v}_{2}$ that determines the exit leaf for $\mathbf{x}$.

Due to the commutative nature of the AND operator, the order in which these aggregations happen does not matter. Because AND is also associative, the overall conjunction of the partial $\mathbf{v}_{w}$ 's will be equivalent to the overall $\mathbf{v}$. As a result of these properties, the blockdistributed Quickscorer will have provably correct predictions.

In our implementation, each server is responsible for a horizontal slice of the data. When a worker pushes a vector of bistrings to a server, the server performs a bitwise AND for each data point. Once all workers that belong to a horizontal data slice have pushed their bitstrings, we can use the aggregated bitstrings on the servers to determine the exit leaves. The workers can finally pull the exit node ids, make the predictions, and prepare to calculate the gradients for the next step.

\subsection{Block-distributed histogram aggregation}

In the block distributed setting each worker will only have a partial view of each data point, so it can only calculate gradients histograms for the data points and range of features it has available. However, since we are performing a simple sum operation over all the gradients, which is a commutative and associative operation, we can perform the local sums at each worker first, push the partial gradients to the servers, and perform the feature-range sum at each server to get the complete histograms for each feature range.
Our communication pattern now changes from the prediction step: each server is now responsible and aggregates the statistics for a range of features instead of data points.

We can think of the gradient histograms as a sparse tensor with dimensions $|L| \times|F| \times B$, where $L$ the set of leaves, $F$ the set of features, and $B$ the number of possible split points. Each worker is responsible for populating a block of this tensor.

For every data point in the block we use the exit nodes from the previous step to get a prediction and gradient value. Given this leaf-to-example mapping, we iterate through each leaf, each data point that belongs to that leaf, and through each feature that belongs to this block. Given the feature index and value, we find the corresponding bucket in the gradient histogram and add the data point's gradient to that bucket, indexed as $(i, j, k)$ where $i$ is the leaf id, $j$ is the feature id, and $k$ is the histogram bucket that corresponds to the feature value.

Once each worker has populated their block of the tensor, they will push their partial tensor to a specific server. Each server is responsible for a range of features, so all workers that have access to the same vertical slice of data will send their data to the same server.

Note that each worker will communicate at most $|L| \times(|F| /|S|) \times B$ elements of the sparse tensor, where $|S|$ the number of servers. However, because we are using a sparse representation, none of the zero values in the histograms will be communicated, making this step potentially much more efficient than existing approaches that always communicate $|L| \times|F| \times B$ elements per worker.

On the server side, every time a new sparse tensor arrives it is simply summed together with the existing one, where the initial element is a tensor of all zeros. Once all workers for a vertical slice have sent their partial sums to their corresponding server, that server will have a complete view of the gradient histograms for the range of features it is responsible for, and we can proceed to the final step of split finding. For this, we use an algorithm similar to the one described in [6], so we omit its description due to space limitations.

\section{EVALUATION}

In this section we present an evaluation of our block-distributed approach in terms of communication efficiency and how that translates to end-to-end runtime.

\subsection{Experimental Setup}

To ensure a fair comparison between the methods we implement both the block-distributed and row-distributed algorithms in $\mathrm{C}^{++}$. We make use to the parameter server originally developed by $\mathrm{Li}$ et al. for the block-distributed implementation and use the Rabit [2] all-reduce framework for the row-distributed version.

We use a local cluster of 12 workers. We use all twelve as workers for the row-distributed experiments, and use 9 as workers and 3 as servers for the block-distributed experiments.

We use four large-scale binary classification datasets ${ }^{1}$ with different sparsity characteristics to evaluate the performance of each approach. The first two, URL and avazu, are extremely sparse with approximately 3.2 million and 1 million features respectively.

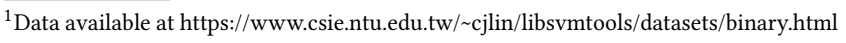




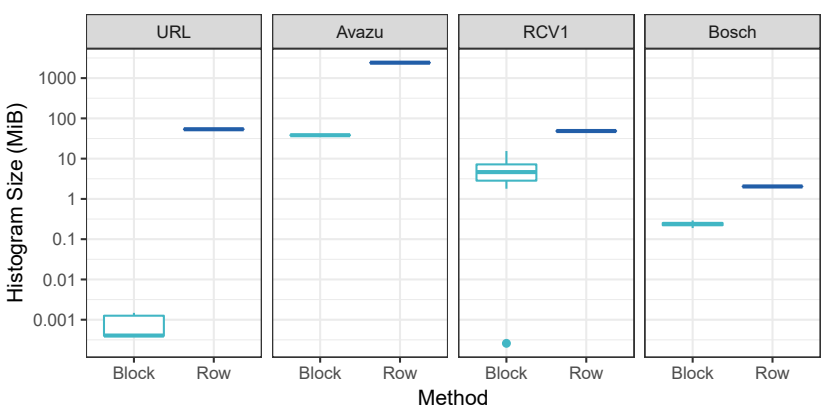

Figure 1: The byte size of the gradient histograms being communicated for the various datasets.

RCV1 has approximately 47 thousand features, and Bosch is a dense dataset with 968 features. Since we focus on feature density we train on a 20,000 data point sample from each dataset.

Our main metric is communication cost, measured in the amount of data the being communicated for the gradient histograms, in MiB. In addition, to measure the real-world performance of the approach we compare the end-to-end runtime as the combined computation and communication time of the histogram aggregation step.

\subsection{Results}

We start with communication cost, illustrated in Figure 1. From the figure, where the $y$-axis is in log scale, we can see the significant communication savings that the sparse histogram representation brings: the histograms produced by the row-distributed algorithms are one to five orders of magnitude larger than the sparse ones developed for this study.

The communication time is therefore significantly reduced for the block-distributed approach, however there is an increase in computation time. This is caused by the overhead introduced by the use of the parameter server and sparse tensors. Unlike dense data structures that are contiguous in memory, the sparse tensors require indirect addressing, resulting in an implementation that is not cache-friendly. In addition, the parameter server approach requires us to pack and unpack the sparse tensors into contiguous arrays of floating point numbers, which are then serialized to be sent over the network and de-serialized server-side. This all creates a significant overhead, as evidenced by our experiments on the less sparse RCV1 and Bosch datasets where the computation phase dominates the communication time, as shown in Figure 2.

\section{CONCLUSIONS}

In this study we presented the first block-distributed algorithm for Gradient Boosted Tree training. Our design allows us to scaleout learning across both the data point and feature dimensions, allowing for cheaper hardware to be used to train massive, sparse datasets. Through our use of a sparse representation of the gradient histograms, we are able to reduce the communication cost of the algorithm by multiple orders of magnitude for sparse data. However, the approach introduces computational overhead that makes it inefficient for datasets with limited sparsity.

In future work we aim to overcome this limitation by using more cache-friendly sparse tensor representations. One benefit of
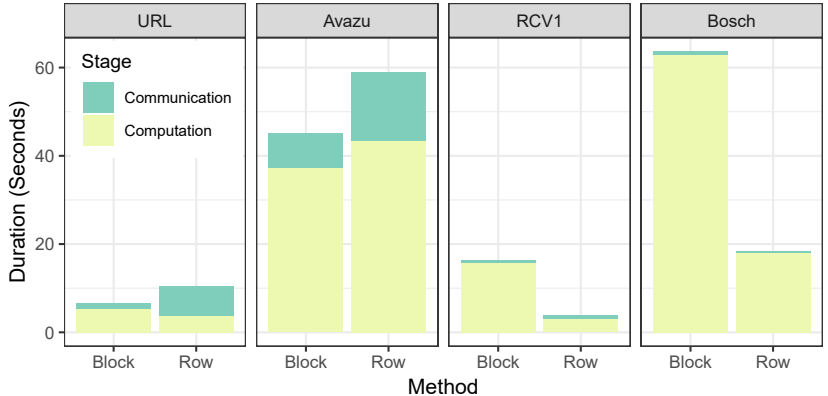

Figure 2: The communication and computation times for the various datasets, in seconds.

the parameter server is the ability to overlap communication with computation, e.g. we could be performing the split finding server side while the gradients are being sent by the workers. Finally, we can make use of the information from previous iterations to prioritize features that more likely to be the best split point, and make use of the Hoeffding bound to stop split finding early.

\section{REFERENCES}

[1] Peter Bühlmann and Torsten Hothorn. 2007. Boosting Algorithms: Regularization, Prediction and Model Fitting. Statist. Sci. 22 (2007), 477-505.

[2] Tianqi Chen, Ignacio Cano, and Tianyi Zhou. 2015. RABIT: A Reliable Allreduce and Broadcast Interface. Technical Report. University of Washington.

[3] Tianqi Chen and Carlos Guestrin. 2016. XGBoost: A Scalable Tree Boosting System. In Proceedings of the 22nd ACM SIGKDD International Conference on Knowledge Discovery and Data Mining (KDD '16). 785-794.

[4] Domenico Dato, Claudio Lucchese, Franco Maria Nardini, Salvatore Orlando, Raffaele Perego, Nicola Tonellotto, and Rossano Venturini. 2016. Fast Ranking with Additive Ensembles of Oblivious and Non-Oblivious Regression Trees. ACM Trans. Inf. Syst. 35, 2 (2016), 15:1-15:31. https://doi.org/10.1145/2987380

[5] Xinran He, Junfeng Pan, Ou Jin, Tianbing Xu, Bo Liu, Tao Xu, Yanxin Shi, Antoine Atallah, Ralf Herbrich, Stuart Bowers, and Joaquin Quiñonero Candela. 2014. Practical Lessons from Predicting Clicks on Ads at Facebook. In Proceedings of the Eighth International Workshop on Data Mining for Online Advertising (ADKDD'14). 5:1-5:9.

[6] Jiawei Jiang, Bin Cui, Ce Zhang, and Fangcheng Fu. 2018. DimBoost: Boosting Gradient Boosting Decision Tree to Higher Dimensions. In Proceedings of the 2018 International Conference on Management of Data (SIGMOD '18). 1363-1376.

[7] Guolin Ke, Qi Meng, Thomas Finley, Taifeng Wang, Wei Chen, Weidong Ma, Qiwei Ye, and Tie-Yan Liu. 2017. LightGBM: A Highly Efficient Gradient Boosting Decision Tree. In Advances in Neural Information Processing Systems 30. 31463154.

[8] Mu Li, David G. Andersen, Jun Woo Park, Alexander J. Smola, Amr Ahmed, Vanja Josifovski, James Long, Eugene J. Shekita, and Bor-Yiing Su. 2014. Scaling Distributed Machine Learning with the Parameter Server. In 11th USENIX Symposium on Operating Systems Design and Implementation (OSDI 14). 583-598.

[9] Mu Li, David G Andersen, Alexander J Smola, and Kai Yu. 2014. Communication Efficient Distributed Machine Learning with the Parameter Server. In Advances in Neural Information Processing Systems 27. 19-27.

[10] Ping Li, Qiang Wu, and Christopher J. Burges. 2008. McRank: Learning to Rank Using Multiple Classification and Gradient Boosting. In Advances in Neural Information Processing Systems 20. 897-904.

[11] Claudio Lucchese, Franco Maria Nardini, Salvatore Orlando, Raffaele Perego, Nicola Tonellotto, and Rossano Venturini. 2015. QuickScorer: A Fast Algorithm to Rank Documents with Additive Ensembles of Regression Trees. In Proceedings of the 38th International ACM SIGIR Conference on Research and Development in Information Retrieval (SIGIR '15). 73-82.

[12] Qi Meng, Guolin Ke, Taifeng Wang, Wei Chen, Qiwei Ye, Zhi-Ming Ma, and Tie-Yan Liu. 2016. A Communication-Efficient Parallel Algorithm for Decision Tree. In Advances in Neural Information Processing Systems 29. 1279-1287.

[13] Liudmila Prokhorenkova, Gleb Gusev, Aleksandr Vorobev, Anna Veronika Dorogush, and Andrey Gulin. 2018. CatBoost: unbiased boosting with categorical features. In Advances in Neural Information Processing Systems 31. 6638-6648.

[14] Rajeev Thakur, Rolf Rabenseifner, and William Gropp. 2005. Optimization of Collective Communication Operations in MPICH. Int. f. High Perform. Comput. Appl. 19, 1 (2005), 49-66. 